\title{
Weighted norm inequalities for maximal singular integrals with nondoubling measures
}

\author{
by \\ Guoen Hu (Zhengzhou) and Dachun Yang (Beijing)
}

\begin{abstract}
Let $\mu$ be a nonnegative Radon measure on $\mathbb{R}^{d}$ which satisfies $\mu(B(x, r))$ $\leq C r^{n}$ for any $x \in \mathbb{R}^{d}$ and $r>0$ and some positive constants $C$ and $n \in(0, d]$. In this paper, some weighted norm inequalities with $A_{p}^{\varrho}(\mu)$ weights of Muckenhoupt type are obtained for maximal singular integral operators with such a measure $\mu$, via certain weighted estimates with $A_{\infty}^{\varrho}(\mu)$ weights of Muckenhoupt type involving the JohnStrömberg maximal operator and the John-Strömberg sharp maximal operator, where $\varrho, p \in[1, \infty)$.
\end{abstract}

1. Introduction. During the last several years, considerable attention has been paid to the study of function spaces with nondoubling measures and boundedness of singular integrals in these spaces; see [10]-[12], [16]-[19], [1]-[3], [6]. Let $\mu$ be a nonnegative Radon measure on $\mathbb{R}^{d}$ which only satisfies the following growth condition: there exist positive constants $C_{0}$ and $n \in(0, d]$ such that for all $x \in \mathbb{R}^{d}$ and $r>0$,

$$
\mu(B(x, r)) \leq C_{0} r^{n},
$$

where $B(x, r)$ is the open ball centered at some point $x \in \mathbb{R}^{d}$ and having radius $r$. The measure $\mu$ in (1.1) is not assumed to satisfy the doubling condition which is a key assumption in the analysis on spaces of homogeneous type. We recall that $\mu$ is said to satisfy the doubling condition if there exists some positive constant $C$ such that $\mu(B(x, 2 r)) \leq C \mu(B(x, r))$ for all $x \in \mathbb{R}^{d}$ and $r>0$. Some important nondoubling measures satisfying (1.1)

2000 Mathematics Subject Classification: 42B20, 43A99.

Key words and phrases: nondoubling measure, singular integral, sharp maximal operator, weight.

The first author is supported by National Natural Science Foundation of China (No. 10671210), and the second author (who is the corresponding author) is supported by National Science Foundation for Distinguished Young Scholars (No. 10425106) and NCET (No. 04-0142) of Ministry of Education of China. Both authors thank the copy editor, Jerzy Trzeciak, for his careful reading of the manuscript and valuable remarks which made this article more readable. 
and the motivation for developing the analysis related to such measures can be found in [20]. We only point out that the analysis with nondoubling measures plays an essential role in solving the long-standing Painlevé problem by Tolsa in [19].

Let $K$ be a $\mu$-locally integrable function on $\mathbb{R}^{d} \times \mathbb{R}^{d} \backslash\{x=y\}$ such that for any $x \neq y$,

$$
|K(x, y)| \leq C|x-y|^{-n},
$$

and for any $x, y, y^{\prime} \in \mathbb{R}^{d}$ with $|x-y| \geq 2\left|y-y^{\prime}\right|$,

$$
\left|K(x, y)-K\left(x, y^{\prime}\right)\right|+\left|K(y, x)-K\left(y^{\prime}, x\right)\right| \leq C \frac{\left|y-y^{\prime}\right|^{\delta}}{|x-y|^{n+\delta}},
$$

where $\delta \in(0,1]$ and $C>0$ are constants. Then the Calderón-Zygmund operator $T$ is formally defined by setting, for any bounded function $f$ with compact support and $x \notin \operatorname{supp}(f d \mu)$,

$$
T f(x)=\int_{\mathbb{R}^{d}} K(x, y) f(y) d \mu(y) .
$$

This integral may not be convergent for many functions. Thus we consider the truncated operator $T_{\varepsilon}$ for $\varepsilon>0$, which is defined by setting, for any bounded function $f$ with compact support and $x \in \mathbb{R}^{d}$,

$$
T_{\varepsilon} f(x)=\int_{|x-y| \geq \varepsilon} K(x, y) f(y) d \mu(y) .
$$

For $p \in(1, \infty)$, we say that $T$ is bounded on $L^{p}(\mu)$ if $T_{\varepsilon}$ is bounded on $L^{p}(\mu)$ with bound independent of $\varepsilon>0$. Also, we say that $T$ is bounded from $L^{1}(\mu)$ to $L^{1, \infty}(\mu)$ if $T_{\varepsilon}$ is bounded from $L^{1}(\mu)$ to $L^{1, \infty}(\mu)$ with bound independent of $\varepsilon>0$. Here and in what follows, for $p \in(0, \infty), L^{p, \infty}(\mu)$ denotes the usual weak- $L^{p}(\mu)$ space defined by

$$
\begin{aligned}
& L^{p, \infty}(\mu)=\{f: f \text { is } \mu \text {-measurable such that } \\
& \left.\qquad\|f\|_{L^{p, \infty}(\mu)}^{p}=\sup _{\lambda>0} \lambda^{p} \mu\left(\left\{x \in \mathbb{R}^{d}:|f(x)|>\lambda\right\}\right)<\infty\right\} .
\end{aligned}
$$

The maximal operator associated with the operator $T$ is defined by setting, for any bounded function $f$ with compact support and $x \in \mathbb{R}^{d}$,

$$
T^{*} f(x)=\sup _{\varepsilon>0}\left|T_{\varepsilon} f(x)\right| .
$$

In his remarkable work [16], Tolsa established the Calderón-Zygmund decomposition associated with the measure as in (1.1), and proved that if $K$ satisfies (1.2) and (1.3), and $T$ is bounded on $L^{2}(\mu)$, then $T$ is also bounded from $L^{1}(\mu)$ to $L^{1, \infty}(\mu)$ and bounded on $L^{p}(\mu)$ for any $p \in(1,2]$. There are many other works concerning the boundedness of the operators $T$ and $T^{*}$ in function spaces with measures satisfying (1.1), e.g. [11], [17] and [1]-[3]. 
The main purpose of this paper is to establish weighted norm inequalities with weights of Muckenhoupt type for the above Calderón-Zygmund operator $T$ and the corresponding maximal operator $T^{*}$. When the measure $\mu$ as in (1.1) satisfies the additional assumption that for any cube $Q$,

$$
\mu(\partial Q)=0,
$$

that is, the faces (or edges) of any cube have $\mu$-measure zero, Orobitg and Pérez [13] proved that if $K$ satisfies (1.2) and (1.3), and if $T$ is bounded on $L^{2}(\mu)$, then $T$ is bounded on $L^{p}(u)$ for $p \in(1, \infty)$ and $u \in A_{p}(\mu)$, where $A_{p}(\mu)$ consists of the weight functions of Muckenhoupt type associated with $\mu$; see Definition 1.1 below. In this paper, we will prove that, without the assumption (1.7), if $K$ satisfies (1.2) and (1.3), and if $T$ is bounded on $L^{2}(\mu)$, then both $T$ and $T^{*}$ are bounded from $L^{p}(u)$ to $L^{p, \infty}(u)$ for any $p \in[1, \infty)$ and $u \in A_{p}^{\varrho}(\mu)$ with $\varrho \in[1, \infty)$. To state the main result, we first recall some definitions and notation.

By a cube $Q \subset \mathbb{R}^{d}$ we mean a closed cube whose sides are parallel to the axes and which is centered at some point of $\operatorname{supp} \mu$, and we denote its side length by $l(Q)$. A $\mu$-measurable function $u$ is said to be a weight if it is nonnegative and $\mu$-locally integrable. The $A_{p}^{\varrho}(\mu)$ weights of Muckenhoupt type in the setting of nondoubling measures were first introduced by Orobitg and Pérez [13] for $\varrho=1$ and by Komori [6] for $\varrho \in[1, \infty)$.

Definition 1.1. Let $\varrho \in[1, \infty), p \in(1, \infty)$ and $p^{\prime}=p /(p-1)$. A weight $u$ is said to be an $A_{p}^{\varrho}(\mu)$ weight if there exists a positive constant $C$ such that for any cube $Q$,

$$
\left(\frac{1}{\mu(\varrho Q)} \int_{Q} u(x) d \mu(x)\right)\left(\frac{1}{\mu(\varrho Q)} \int_{Q} u(x)^{1-p^{\prime}} d \mu(x)\right)^{p-1} \leq C .
$$

Also, a weight $u$ is said to be an $A_{1}^{\varrho}(\mu)$ weight if there exists a positive constant $C$ such that for any cube $Q$,

$$
\frac{1}{\mu(\varrho Q)} \int_{Q} u(x) d \mu(x) \leq C \inf _{y \in Q} u(y) .
$$

As in the classical setting, we set $A_{\infty}^{\varrho}(\mu)=\bigcup_{p=1}^{\infty} A_{p}^{\varrho}(\mu)$. For $\varrho=1$, we denote $A_{p}^{\varrho}(\mu), A_{1}^{\varrho}(\mu)$ and $A_{\infty}^{\varrho}(\mu)$ simply by $A_{p}(\mu), A_{1}(\mu)$ and $A_{\infty}(\mu)$, respectively.

Our main result is the following weighted weak type estimate for the operator $T^{*}$.

Theorem 1.1. Let $\varrho \in[1, \infty)$ be fixed. Let $K$ satisfy (1.2) and (1.3), and $T$ be the Calderón-Zygmund operator formally defined as in (1.4). If $T$ is bounded on $L^{2}(\mu)$, then for any $p \in[1, \infty)$ and $u \in A_{p}^{o}(\mu)$, the maximal operator $T^{*}$ defined by (1.6) is bounded from $L^{p}(u)$ to $L^{p, \infty}(u)$, that is, there 
exists a positive constant $C$ such that for any $\lambda>0$ and bounded function $f$ with compact support,

$$
u\left(\left\{x \in \mathbb{R}^{d}: T^{*} f(x)>\lambda\right\}\right) \leq C \lambda^{-p} \int_{\mathbb{R}^{d}}|f(x)|^{p} u(x) d \mu(x),
$$

where, for a weight $w$ and a $\mu$-measurable set $E, w(E)=\int_{E} w(x) d \mu(x)$.

By Corollary 2.5(i) in [13], if $\mu$ satisfies the assumption (1.7), then for any $p \in(1, \infty)$ and $u \in A_{p}(\mu), u \in A_{p-\sigma}(\mu)$ for some $\sigma>0$. Thus, Theorem 1.1 along with the Marcinkiewicz interpolation theorem shows that for any $p \in(1, \infty)$ and $u \in A_{p}(\mu), T^{*}$ (and therefore $T$ ) is bounded on $L^{p}(u$ ). This recovers the weighted estimate (11) in [13].

To establish their weighted estimate for the operator $T$ when $\mu$ satisfies (1.7), Orobitg and Pérez in [13] used a distributional inequality involving the singular integral operator and the central Hardy-Littlewood maximal operator, and the fact that for $u \in A_{p}(\mu), p \in(1, \infty)$ and any bounded function $f$ with compact support, $T^{*} f \in L^{p}(u)$. Checking the argument used in [13], we see that this a priori estimate can be replaced by the statement that for $u \in A_{p}(\mu), p \in(1, \infty)$ and any bounded function $f$ with compact support,

$$
T^{*} f \in L^{p, \infty}(u) .
$$

On the other hand, as pointed out by Orobitg and Pérez [13], without the assumption (1.7), the reverse Hölder inequality, the fact that $u \in A_{1}(\mu)$ implies $u \in L_{\text {loc }}^{1+\sigma}(\mu)$ with some $\sigma \in(0, \infty)$, and some other important properties enjoyed by the $A_{p}$ weights in the setting of Euclidean spaces, may not be true; therefore, without (1.7), the a priori estimate (1.8) cannot be verified directly. To avoid (1.7), in the proof of Theorem 1.1, we will establish a distributional inequality linking the John-Strömberg maximal operator and the John-Strömberg sharp maximal operator (see Theorem 2.1 below), which, together with a fairly weak a priori estimate (see (3.10) below), leads to the desired conclusion of Theorem 1.1.

We should point out that Theorem 2 of [9] indicates that for any nonnegative Radon measure $\mu$, there exists an orthonormal system in $\mathbb{R}^{d}$ so that (1.7) holds. However, it is not clear how the $A_{p}(\mu)$ weights and singular integrals related to $\mu$ as in (1.1) depend on different orthonormal systems in $\mathbb{R}^{d}$.

Also, it is obvious that if $1 \leq \varrho_{1}<\varrho_{2}<\infty$, then $A_{p}^{\varrho_{1}}(\mu) \subset A_{p}^{\varrho_{2}}(\mu)$. However, it is still unclear if this inclusion is proper.

Finally, we make some conventions. Throughout the paper, $C$ denotes a positive constant that is independent of the main parameters involved, but whose value may vary from line to line. Constants with subscript, such as $C_{1}$, do not change in different occurrences. Let $\alpha$ and $\beta$ be positive constants such that $\beta>\alpha^{n}$. For a cube $Q$, we say that $Q$ is $(\alpha, \beta)$-doubling if 
$\mu(\alpha Q) \leq \beta \mu(Q)$, where $\alpha Q$ denote the cube concentric with $Q$ and having side length $\alpha l(Q)$. It was pointed out by Tolsa [17] that there exists a large constant $\beta=\beta_{\alpha, d}>0$ such that for any $x \in \operatorname{supp} \mu$ and $R>0$, there exists some $\left(\alpha, \beta_{\alpha, d}\right)$-doubling cube centered at $x$ with $l(Q)>R$, and for $\mu$-almost all $x \in \mathbb{R}^{d}$, there exists a sequence $\left\{Q_{k}\right\}_{k \in \mathbb{N}}$ of $\left(\alpha, \beta_{\alpha, d}\right)$-doubling cubes centered at $x$ with $l\left(Q_{k}\right) \rightarrow 0$ as $k \rightarrow \infty$. In what follows, for a fixed $\varrho \in[1, \infty)$, by a doubling cube $Q$, we always mean that $Q$ is a $\left(2 \varrho, \beta_{2 \varrho, d}\right)$-doubling cube. Moreover, for a cube $Q, \widetilde{Q}$ denotes the smallest doubling cube of the form $(2 \varrho)^{k} Q$ with $k \in \mathbb{N} \cup\{0\}$.

2. John-Strömberg maximal operators. The purpose of this section is to introduce the John-Strömberg maximal operator and the JohnStrömberg sharp maximal operator related to the measure in (1.1), and then establish certain weighted norm inequalities with $A_{\infty}^{\varrho}(\mu)$-weights relating these two operators, where $\varrho \in[1, \infty)$. This weighted estimate plays an important role in the proof of Theorem 1.1 and is of independent interest.

For a cube $Q$ with $\mu(Q) \neq 0$, and a real-valued locally integrable function $f, m_{f}(Q)$, the median value of $f$ on the cube $Q$, is defined to be any number such that

$$
\begin{aligned}
& \mu\left(\left\{y \in Q: f(y)>m_{f}(Q)\right\}\right) \leq \frac{1}{2} \mu(Q), \\
& \mu\left(\left\{y \in Q: f(y)<m_{f}(Q)\right\}\right) \leq \frac{1}{2} \mu(Q) .
\end{aligned}
$$

If $\mu(Q)=0$, we set $m_{f}(Q)=0$ for any real-valued $\mu$-locally integrable function $f$. If $f$ is complex-valued, the median value of $f$ is defined by $m_{f}(Q)=m_{\operatorname{Re}(f)}(Q)+i m_{\operatorname{Im}(f)}(Q)$, where $i^{2}=-1$.

Let $\varrho \in[1, \infty)$ and $s \in\left(0, \beta_{2 \varrho, d}^{-1} / 4\right)$. For any fixed cube $Q$ and $\mu$-locally integrable function $f$, define $m_{0, s ; Q}^{\varrho}(f)$ by

$$
m_{0, s ; Q}^{\varrho}(f)=\inf \left\{t>0: \mu(\{y \in Q:|f(y)|>t\})<s \mu\left(\frac{3}{2} \varrho Q\right)\right\}
$$

when $\mu(Q) \neq 0$, and $m_{0, s ; Q}^{\varrho}(f)=0$ when $\mu(Q)=0$. The John-Strömberg maximal operator $M_{0, s}^{\rho, d}$ is defined by setting, for all $x \in \mathbb{R}^{d}$,

$$
M_{0, s}^{\varrho, d} f(x)=\sup _{Q \ni x, Q \text { doubling }} m_{0, s ; Q}^{\varrho}(f) .
$$

For any two cubes $Q_{1} \subset Q_{2}$, set

$$
\delta_{Q_{1}, Q_{2}}^{\varrho}=1+\sum_{k=1}^{N_{Q_{1}, Q_{2}}^{\varrho}} \frac{\mu\left((2 \varrho)^{k} Q_{1}\right)}{\left[l\left((2 \varrho)^{k} Q_{1}\right)\right]^{n}},
$$

where $N_{Q_{1}, Q_{2}}^{\varrho}$ is the least positive integer $k$ such that $l\left((2 \varrho)^{k} Q_{1}\right) \geq l\left(Q_{2}\right)$. The John-Strömberg sharp maximal operator $M_{0, s}^{\varrho, \sharp}$ is defined by setting, for 
all $x \in \mathbb{R}^{d}$,

$$
M_{0, s}^{\varrho, \sharp} f(x)=\sup _{Q \ni x} m_{0, s ; Q}^{\varrho}\left(f-m_{f}(\widetilde{Q})\right)+\sup _{\substack{x \in Q \subset R \\ Q, R \text { doubling }}} \frac{\left|m_{f}(Q)-m_{f}(R)\right|}{\delta_{Q, R}^{\varrho}} .
$$

The operators $M_{0, s}^{\varrho, d}$ and $M_{0, s}^{\varrho, \sharp}$ in the setting of Euclidean spaces were first introduced by John [5] and then rediscovered by Strömberg [15] and Lerner $[7,8]$. It is easy to verify that for any cube $Q \ni x$ and $\varepsilon>0$,

$$
\mu\left(\left\{y \in Q:\left|f(y)-m_{f}(\widetilde{Q})\right|>M_{0, s}^{\varrho, \sharp} f(x)+\varepsilon\right\}\right)<s \mu\left(\frac{3}{2} \varrho Q\right) .
$$

Our main result in this section is the following theorem.

Theorem 2.1. Let $\varrho \in[1, \infty), s_{1} \in\left(0, \beta_{2 \varrho, d}^{-1} / 4\right), p \in(0, \infty)$ and $u \in$ $A_{\infty}^{\varrho}(\mu)$. Then there exist a constant $C_{1} \in(0,1)$, depending on $s_{1}$ and $u$, and a positive constant $C$ such that for any $s_{2} \in\left(0, C_{1} s_{1}\right)$,

(i) if $\mu\left(\mathbb{R}^{d}\right)=\infty, f \in L^{p_{0}, \infty}(\mu)$ with $p_{0} \in[1, \infty)$, and for any $R>0$,

$$
\sup _{0<\lambda<R} \lambda^{p} u\left(\left\{x \in \mathbb{R}^{d}:|f(x)|>\lambda\right\}\right)<\infty
$$

then

$$
\begin{aligned}
\sup _{\lambda>0} \lambda^{p} u\left(\left\{x \in \mathbb{R}^{d}: M_{0, s_{1}}^{\varrho, d} f(x)>\lambda\right\}\right) & \\
& \leq C \sup _{\lambda>0} \lambda^{p} u\left(\left\{x \in \mathbb{R}^{d}: M_{0, s_{2}}^{\varrho, \sharp} f(x)>\lambda\right\}\right) ;
\end{aligned}
$$

(ii) if $\mu\left(\mathbb{R}^{d}\right)<\infty$ and $f \in L^{p_{0}, \infty}(\mu)$ with $p_{0} \in[1, \infty)$, then

$$
\begin{aligned}
\sup _{\lambda>0} \lambda^{p} u\left(\left\{x \in \mathbb{R}^{d}: M_{0, s_{1}}^{\varrho, d}\right.\right. & f(x)>\lambda\}) \\
\leq & C \sup _{\lambda>0} \lambda^{p} u\left(\left\{x \in \mathbb{R}^{d}: M_{0, s_{2}}^{\varrho, \sharp} f(x)>\lambda\right\}\right) \\
& +C u\left(\mathbb{R}^{d}\right)\left(s_{1} \mu\left(\mathbb{R}^{d}\right)\right)^{-p / p_{0}}\|f\|_{L^{p_{0}, \infty}(\mu)}^{p} .
\end{aligned}
$$

To prove Theorem 2.1, we first give some preliminary results about $A_{p}^{\varrho}(\mu)$.

Let $\varrho \in[1, \infty)$ be fixed. For $\eta \in(1, \infty)$, define the maximal operator $M_{\eta}$ by setting, for all $x \in \mathbb{R}^{d}$,

$$
M_{\eta} f(x)=\sup _{Q \ni x} \frac{1}{\mu(\eta Q)} \int_{Q}|f(y)| d \mu(y) .
$$

A result of Komori [6] states that for any $\eta>\varrho, p \in[1, \infty)$ and $u \in A_{p}^{\varrho}(\mu)$, $M_{\eta}$ is bounded from $L^{p}(u)$ to $L^{p, \infty}(u)$. Let $M^{\varrho, d}$ be the doubling maximal operator defined by setting, for all $x \in \mathbb{R}^{d}$,

$$
M^{\varrho, d} f(x)=\sup _{Q \ni x, Q \text { doubling }} \frac{1}{\mu(Q)} \int_{Q}|f(y)| d \mu(y) .
$$


Notice that for any doubling cube $Q$,

$$
\frac{1}{\mu(Q)} \int_{Q}|f(y)| d \mu(y) \leq \beta_{2 \varrho, d} \frac{1}{\mu(2 \varrho Q)} \int_{Q}|f(y)| d \mu(y) \leq C \inf _{x \in Q} M_{2 \varrho} f(x) .
$$

Therefore, we have the following conclusion.

Lemma 2.1. Let $\varrho \in[1, \infty)$ and let $M_{\eta}$ and $M^{\varrho, d}$ be the maximal operators defined by (2.5) and (2.6), respectively. For any $p \in[1, \infty)$ and $u \in A_{p}^{\varrho}(\mu)$, both $M_{\eta}$ with $\eta \in(\varrho, \infty)$ and $M^{\varrho, d}$ are bounded from $L^{p}(u)$ to $L^{p, \infty}(u)$.

As an easy consequence of Lemma 2.1, we obtain the following result.

Lemma 2.2. Let $\varrho, p \in[1, \infty), u \in A_{p}^{\varrho}(\mu)$ and $\eta \in(\varrho, \infty)$. Then there exist constants $C_{2}, C_{3} \geq 1$ such that

(i) for any cube $Q$ and $\mu$-measurable set $E \subset Q$,

$$
\frac{u(E)}{u(Q)} \geq C_{2}^{-1}\left(\frac{\mu(E)}{\mu(\eta Q)}\right)^{p}
$$

(ii) for any doubling cube $Q$ and $\mu$-measurable set $E \subset Q$,

$$
\frac{u(E)}{u(Q)} \geq C_{3}^{-1}\left(\frac{\mu(E)}{\mu(Q)}\right)^{p}
$$

(iii) for any doubling cube $Q$ and $\mu$-measurable set $E \subset Q$,

$$
\frac{u(E)}{u(Q)} \leq 1-C_{3}^{-1}\left(1-\frac{\mu(E)}{\mu(Q)}\right)^{p} .
$$

Proof. Obviously, (ii) follows from (i), and (iii) is an easy consequence of (ii) with $E$ replaced by $Q \backslash E$. So it suffices to prove (i), whose proof is similar to that of the classical case; see [14]. In fact, it is easy to see that for any cube $Q$ and any $\mu$-measurable set $E \subset Q$,

$$
\inf _{x \in Q} M_{\eta} \chi_{E}(x) \geq \mu(E) / \mu(\eta Q) .
$$

On the other hand, Lemma 2.1 states that for any $p \in[1, \infty), u \in A_{p}^{\varrho}(\mu)$ and $\eta>\varrho$, there exists a constant $C_{2} \geq 1$ such that for any $\lambda>0$,

$$
u\left(\left\{x \in \mathbb{R}^{d}: M_{\eta} f(x)>\lambda\right\}\right) \leq C_{2} \lambda^{-p} \int_{\mathbb{R}^{d}}|f(x)|^{p} u(x) d \mu(x) .
$$

Thus, both estimates imply that for any $\lambda \in(0, \mu(E) / \mu(\eta Q))$,

$$
u(Q) \leq u\left(\left\{x \in \mathbb{R}^{d}: M_{\eta} \chi_{E}(x)>\lambda\right\}\right) \leq C_{2} \lambda^{-p} u(E),
$$

and so, for any $\lambda<\mu(E) / \mu(\eta Q)$,

$$
\frac{u(E)}{u(Q)} \geq C_{2}^{-1} \lambda^{p} .
$$


The conclusion (i) follows by letting $\lambda \rightarrow \mu(E) / \mu(\eta Q)$, which completes the proof of Lemma 2.2.

Lemma 2.3. Let $\varrho, p \in[1, \infty)$ and $s \in\left(0, \beta_{2 \varrho, d}^{-1} / 4\right)$. Then for all $\mu$-locally integrable functions $f$ and $\lambda>0$,

(i) $\left\{x \in \mathbb{R}^{d}:|f(x)|>\lambda\right\} \subset\left\{x \in \mathbb{R}^{d}: M_{0, s}^{\varrho, d} f(x) \geq \lambda\right\} \cup \Theta$ with $\mu(\Theta)=0$;

(ii) for $u \in A_{p}^{\varrho}(\mu)$,

$$
u\left(\left\{x \in \mathbb{R}^{d}: M_{0, s}^{\varrho, d} f(x)>\lambda\right\}\right) \leq C s^{-p} u\left(\left\{x \in \mathbb{R}^{d}:|f(x)|>\lambda\right\}\right),
$$

where $C$ is a positive constant depending on $d$ and $\varrho$, but not on $s$ and the weight $u$.

Proof. By the Lebesgue differential theorem, we know that for $\mu$-almost all $x \in \mathbb{R}^{d}$,

$$
|f(x)| \leq M^{\varrho, d} f(x)
$$

and so

$$
\begin{aligned}
\left\{x \in \mathbb{R}^{d}:|f(x)|\right. & >\lambda\} \\
= & \left\{x \in \mathbb{R}^{d}: \chi_{\left\{y \in \mathbb{R}^{d}:|f(y)|>\lambda\right\}}(x)=1\right\} \\
& \subset\left\{x \in \mathbb{R}^{d}: M^{\varrho, d}\left(\chi_{\left\{y \in \mathbb{R}^{d}:|f(y)|>\lambda\right\}}\right)(x)>s \beta_{2 \varrho, d}\right\} \cup \Theta,
\end{aligned}
$$

where $\mu(\Theta)=0$. On the other hand, if $M^{\varrho, d}\left(\chi_{\left\{y \in \mathbb{R}^{d}:|f(y)|>\lambda\right\}}\right)(x)>s \beta_{2 \varrho, d}$, then there exists a doubling cube $Q$ containing $x$ such that $\mu(Q)>0$ and

$$
\mu(\{y \in Q:|f(y)|>\lambda\})>s \beta_{2 \varrho, d} \mu(Q) \geq s \mu\left(\frac{3}{2} \varrho Q\right) .
$$

Notice that for any $t>m_{0, s ; Q}^{\varrho}(f)$,

$$
\mu(\{y \in Q:|f(y)|>t\})<s \mu\left(\frac{3}{2} \varrho Q\right) .
$$

Thus $m_{0, s ; Q}^{\varrho}(f) \geq \lambda$, and so $M_{0, s}^{\varrho, d} f(x) \geq \lambda$, which yields (i).

We now turn to (ii). For any fixed $\lambda, r>0$ and $x \in \mathbb{R}^{d}$, set

$$
M_{0, s}^{\varrho, d, r} f(x)=\sup _{Q \ni x, l(Q)<r, Q \text { doubling }} m_{0, s ; Q}^{\varrho}(f)
$$

and

$$
E_{r, \lambda}=\left\{x \in \mathbb{R}^{d}: M_{0, s}^{\varrho, d, r} f(x)>\lambda\right\} .
$$

For any $x \in E_{r, \lambda}$, there exists a doubling cube $Q_{x}$ such that $x \in Q_{x}$, $l\left(Q_{x}\right)<r$, and

$$
\mu\left(\left\{y \in Q_{x}:|f(y)|>\lambda\right\}\right) \geq s \mu\left(\frac{3}{2} \varrho Q_{x}\right) .
$$

It now follows from Lemma 2.2(ii) that

$$
u\left(\left\{y \in Q_{x}:|f(y)|>\lambda\right\}\right) \geq C s^{p} u\left(\frac{3}{2} \varrho Q_{x}\right) .
$$


By the Besicovitch covering lemma, we obtain a family $\left\{Q_{\tau}\right\}_{\tau} \subset\left\{Q_{x}\right\}_{x \in E_{r, \lambda}}$ of cubes such that

$$
E_{r, \lambda} \subset \bigcup_{\tau} 2 \varrho Q_{\tau}, \quad \sum_{\tau} \chi_{Q_{\tau}} \leq C_{d},
$$

where $C_{d}$ is a positive integer depending only on $d$. Therefore,

$$
\begin{aligned}
u\left(E_{r, \lambda}\right) & \leq C s^{-p} \sum_{\tau} u\left(\left\{y \in Q_{\tau}:|f(y)|>\lambda\right\}\right) \\
& \leq C s^{-p} u\left(\left\{y \in \mathbb{R}^{d}:|f(y)|>\lambda\right\}\right),
\end{aligned}
$$

which together with a certain basic property of measures proves (ii). This completes the proof of Lemma 2.3.

Lemma 2.4. Let $\varrho \in[1, \infty), s \in\left(0, \beta_{2 \varrho, d}^{-1} / 4\right)$ and $Q$ be a doubling cube with $\mu(Q) \neq 0$. For any constant $c \in \mathbb{C}$ and $\mu$-locally integrable function $f$,

$$
\left|m_{0, s ; Q}^{\varrho}(f)-\right| c|| \leq m_{0, s ; Q}^{\varrho}(f-c) .
$$

Proof. It suffices to prove that

$$
m_{0, s ; Q}^{\varrho}(f)+m_{0, s ; Q}^{\varrho}(f-c) \geq|c|, \quad m_{0, s ; Q}^{\varrho}(f) \leq m_{0, s ; Q}^{\varrho}(f-c)+|c| .
$$

To verify the first inequality, we observe that if $t_{1}, t_{2}>0$ satisfy

$$
\begin{array}{r}
\mu\left(\left\{y \in Q:|f(y)-c|>t_{1}\right\}\right)<s \mu\left(\frac{3}{2} \varrho Q\right), \\
\mu\left(\left\{y \in Q:|f(y)|>t_{2}\right\}\right)<s \mu\left(\frac{3}{2} \varrho Q\right),
\end{array}
$$

then $t_{1}+t_{2} \geq|c|$. Otherwise,

$$
\begin{aligned}
\mu\left(\left\{y \in Q:|f(y)| \leq t_{2}\right\}\right) & \leq \mu\left(\left\{y \in Q:|f(y)-c|>t_{1}\right\}\right) \\
& <s \mu\left(\frac{3}{2} \varrho Q\right)<\frac{1}{2} \mu(Q),
\end{aligned}
$$

which contradicts the fact that

$$
\mu\left(\left\{y \in Q:|f(y)| \leq t_{2}\right\}\right)>\mu(Q)-s \mu\left(\frac{3}{2} \varrho Q\right) \geq \frac{1}{2} \mu(Q) .
$$

Therefore,

$$
m_{0, s ; Q}^{\varrho}(f)+m_{0, s ; Q}^{\varrho}(f-c) \geq|c| .
$$

On the other hand, for any $\varepsilon>0$, we can take $t_{0}>0$ such that $t_{0}<$ $m_{0, s ; Q}^{\varrho}(f-c)+\varepsilon$, and that

$$
\mu\left(\left\{y \in Q:|f(y)-c|>t_{0}\right\}\right)<s \mu\left(\frac{3}{2} \varrho Q\right) .
$$

This in turn implies that

$$
\mu\left(\left\{y \in Q:|f(y)|>t_{0}+|c|\right\}\right)<s \mu\left(\frac{3}{2} \varrho Q\right) .
$$

Thus,

$$
m_{0, s ; Q}^{\varrho}(f) \leq t_{0}+|c|<m_{0, s ; Q}^{\varrho}(f-c)+|c|+\varepsilon,
$$

which implies the desired estimate by letting $\varepsilon \rightarrow 0$, and hence completes the proof of Lemma 2.4. 
Lemma 2.5. Let $\varrho, p \in[1, \infty), s \in\left(0, \beta_{2 \varrho, d}^{-1} / 4\right)$ and $Q$ be a doubling cube. Then for any $\mu$-locally integrable real-valued function $f$,

$$
\left|m_{f}(Q)\right| \leq m_{0, s ; Q}^{\varrho}(f) .
$$

Proof. Without loss of generality, we may assume that $\mu(Q)>0$. If $f$ is real-valued and $m_{f}(Q) \geq 0$, we have

$$
\begin{aligned}
\{y \in Q:|f(y)| & \left.\geq\left|m_{f}(Q)\right|\right\} \\
& =\left\{y \in Q: f(y) \geq m_{f}(Q)\right\} \cup\left\{y \in Q: f(y) \leq-m_{f}(Q)\right\} ;
\end{aligned}
$$

and if $m_{f}(Q)<0$,

$$
\begin{aligned}
\{y \in Q:|f(y)| & \left.\geq\left|m_{f}(Q)\right|\right\} \\
= & \left\{y \in Q: f(y) \geq-m_{f}(Q)\right\} \cup\left\{y \in Q: f(y) \leq m_{f}(Q)\right\} .
\end{aligned}
$$

Therefore, by the definition of $m_{f}(Q)$,

$$
\begin{aligned}
& \mu\left(\left\{y \in Q:|f(y)| \geq\left|m_{f}(Q)\right|\right\}\right) \\
& \quad \geq \min \left\{\mu\left(\left\{y \in Q: f(y) \geq m_{f}(Q)\right\}\right), \mu\left(\left\{y \in Q: f(y) \leq m_{f}(Q)\right\}\right)\right\} \\
& \quad \geq \frac{1}{2} \mu(Q) .
\end{aligned}
$$

This implies that for any $t>0$ satisfying

$$
\mu(\{y \in Q:|f(y)|>t\})<s \mu\left(\frac{3}{2} \varrho Q\right),
$$

we have $t \geq\left|m_{f}(Q)\right|$. Otherwise, we obtain

$$
\mu\left(\left\{y \in Q:|f(y)| \geq\left|m_{f}(Q)\right|\right\}\right)<s \mu(2 \varrho Q) \leq s \beta_{2 \varrho, d} \mu(Q)<\frac{1}{4} \mu(Q),
$$

which contradicts (2.7). Our desired result now follows directly by taking the infimum over $t$, which completes the proof of Lemma 2.5.

Lemma 2.6. Let $\varrho \in[1, \infty), s_{1} \in\left(0, \beta_{2 \varrho, d}^{-1} / 4\right)$ and $u \in A_{p}^{\varrho}(\mu)$ for some $p \in[1, \infty)$. Then there exists a constant $C_{4} \in(1, \infty)$ such that for any $s_{2} \in\left(0, C_{4}^{-1} s_{1}\right), \gamma>0$ and real-valued function $f \in L^{p_{0}, \infty}(\mu)$ with some $p_{0} \in[1, \infty)$,

$$
\begin{aligned}
u\left(\left\{x \in \mathbb{R}^{d}: M_{0, s_{1}}^{\varrho, d} f(x)>(1+\gamma) \lambda,\right.\right. & \left.\left.M_{0, s_{2}}^{\varrho, \sharp} f(x) \leq \theta_{2} \gamma \lambda\right\}\right) \\
& \leq \theta_{1} u\left(\left\{x \in \mathbb{R}^{d}: M_{0, s_{1}}^{\varrho, d} f(x)>\lambda\right\}\right),
\end{aligned}
$$

provided that

(i) $\mu\left(\mathbb{R}^{d}\right)=\infty$ and $\lambda>0$, or

(ii) $\mu\left(\mathbb{R}^{d}\right)<\infty$ and $\lambda>\lambda_{f}=\left(s_{1} \mu\left(\mathbb{R}^{d}\right)\right)^{-1 / p_{0}}\|f\|_{L^{p_{0}, \infty}(\mu)}$,

where $\theta_{1}, \theta_{2} \in(0,1)$ are constants depending only on $d, \varrho$ and $\mu$. 
Proof. Let $\lambda_{f}=0$ if $\mu\left(\mathbb{R}^{d}\right)=\infty$ and $\lambda_{f}=\left(s_{1} \mu\left(\mathbb{R}^{d}\right)\right)^{-1 / p_{0}}\|f\|_{L^{p_{0}, \infty}(\mu)}$ if $\mu\left(\mathbb{R}^{d}\right)<\infty$. For each fixed $\lambda>\lambda_{f}$, set

$$
\begin{aligned}
& \Omega_{\lambda}=\left\{x \in \mathbb{R}^{d}: M_{0, s_{1}}^{\varrho, d} f(x)>\lambda\right\}, \\
& E_{\lambda}=\left\{x \in \mathbb{R}^{d}: M_{0, s_{1}}^{\varrho, d} f(x)>(1+\gamma) \lambda, M_{0, s_{2}}^{\varrho, \sharp} f(x) \leq \theta_{2} \gamma \lambda\right\} .
\end{aligned}
$$

Notice that if $\lambda>\lambda_{f}$, then

$$
\mu\left(\left\{y \in \mathbb{R}^{d}:|f(y)|>\lambda\right\}\right) \leq \frac{\|f\|_{L^{p_{0}, \infty}(\mu)}^{p_{0}}}{\lambda^{p_{0}}}<s_{1} \mu\left(\mathbb{R}^{d}\right),
$$

and so

$$
\frac{1}{\mu\left(\mathbb{R}^{d}\right)} \int_{\left\{y \in \mathbb{R}^{d}:|f(y)|>\lambda\right\}} d \mu(y)<s_{1} .
$$

Moreover, as pointed out in Section 1 , for $\mu$-almost all $x \in \mathbb{R}^{d}$, there exists a sequence $\left\{I_{k}\right\}_{k}$ of doubling cubes with $l\left(I_{k}\right) \rightarrow \infty$ as $k \rightarrow \infty$. Therefore, by the basic property of $\mu$, we may assume that for any $\lambda>\lambda_{f}$ and any $x \in \mathbb{R}^{d}$,

$$
\lim _{I \ni x, l(I) \rightarrow \infty, I \text { doubling }} \frac{1}{\mu(I)} \int_{\{y \in I:|f(y)|>\lambda\}} d \mu(y)<s_{1},
$$

which implies that

$$
\lim _{I \ni x, l(I) \rightarrow \infty, I \text { doubling }} m_{0, s_{1} ; I}^{\varrho}(f)<\lambda .
$$

On the other hand, for each fixed $x \in E_{\lambda}$, there is a doubling cube $Q$ containing $x$ such that $m_{0, s_{1} ; Q}^{\varrho}(f)>(1+\gamma / 2) \lambda$. The inequality (2.8) tells us that among these doubling cubes, there exists one doubling cube, denoted by $Q_{x}$, which has almost maximal side length, in the sense that if some doubling cube $I$ contains $x$ and has side length no less than $2 \varrho l\left(Q_{x}\right)$, then $m_{0, s_{1} ; I}^{\varrho}(f) \leq(1+\gamma / 2) \lambda$; see also [17, p. 128].

Let $R_{x}$ be the cube centered at $x$ with side length $3 \varrho l\left(Q_{x}\right)$, and set $S_{x}=\widetilde{R}_{x}$. An application of Lemma 2.4 gives

$$
\begin{aligned}
\mid m_{0, s_{1} ; Q_{x}}^{\varrho}(f)- & m_{0, s_{1} ; S_{x}}^{\varrho}(f) \mid \\
\leq & \left|m_{0, s_{1} ; Q_{x}}^{\varrho}(f)-\right| m_{f}\left(Q_{x}\right)|| \\
& +\left|m_{f}\left(Q_{x}\right)-m_{f}\left(S_{x}\right)\right|+\left|m_{0, s_{1} ; S_{x}}^{\varrho}(f)-\right| m_{f}\left(S_{x}\right)|| \\
\leq & m_{0, s_{1} ; Q_{x}}^{\varrho}\left(f-m_{f}\left(Q_{x}\right)\right) \\
& +\left|m_{f}\left(Q_{x}\right)-m_{f}\left(S_{x}\right)\right|+m_{0, s_{1} ; S_{x}}^{\varrho}\left(f-m_{f}\left(S_{x}\right)\right) \\
\leq & 3 \delta_{Q_{x}, S_{x}}^{\varrho} \inf _{y \in Q_{x}} M_{0, s_{1}}^{\varrho, \sharp} f(y) \leq 3 \delta_{Q_{x}, S_{x}}^{\varrho} M_{0, s_{2}}^{\varrho, \sharp} f(x) \leq C_{5} \theta_{2} \gamma \lambda,
\end{aligned}
$$

where $C_{5}$ is a positive constant depending only on $d, \varrho$ and $\mu$ such that 
$3 \delta_{Q_{x}, S_{x}}^{\varrho} \leq C_{5}$; see Lemma 2.1 in [17]. If we choose $\theta_{2}>0$ small enough, it follows that $m_{0, s_{1} ; S_{x}}^{\varrho}(f)>\lambda$, and so $S_{x} \subset \Omega_{\lambda}$.

By the Besicovitch covering lemma, we see that there exist $n_{B}$ subfamilies $\mathcal{D}_{k}=\left\{S_{j}^{k}\right\}_{j}, k=1, \ldots, n_{B}$, of cubes $\left\{S_{x}\right\}_{x \in E_{\lambda}}$ such that

(i) $E_{\lambda} \subset \bigcup_{k=1}^{n_{B}} \bigcup_{j} S_{j}^{k}$, and for each $j$ and $k, m_{0, s_{1} ; S_{j}^{k}}^{\varrho}(f)>\lambda$;

(ii) for each subfamily $\mathcal{D}_{k}\left(1 \leq k \leq n_{B}\right)$, the cubes in $\mathcal{D}_{k}$ are pairwise disjoint;

(iii) each cube $S_{j}^{k}$ is doubling and centered at some point $x_{j}^{k} \in E_{\lambda}$.

We can obtain at least one family, which without loss of generality is supposed to be $\mathcal{D}_{1}$, such that

$$
u\left(\bigcup_{j} S_{j}^{1}\right) \geq n_{B}^{-1} u\left(\bigcup_{j, k} S_{j}^{k}\right) .
$$

If we can prove that there exists a positive constant $C_{6}$ such that for each $S_{j}^{1} \in \mathcal{D}_{1}$,

$$
\mu\left(S_{j}^{1} \cap E_{\lambda}\right) \leq C_{6} s_{1}^{-1} s_{2} \mu\left(S_{j}^{1}\right),
$$

then it follows from Lemma 2.2 that

$$
u\left(S_{j}^{1} \cap E_{\lambda}\right) \leq\left(1-C_{3}^{-1}\left(1-C_{6} s_{1}^{-1} s_{2}\right)^{p}\right) u\left(S_{j}^{1}\right) .
$$

Let $C_{4}=1+C_{6}$ and $C_{7}=1-C_{3}^{-1}\left(1-C_{6} s_{1}^{-1} s_{2}\right)^{p}$. Recall that $\left\{S_{j}^{1}\right\}_{j}$ are pairwise disjoint. Thus, for $s_{2} \in\left(0, C_{4}^{-1} s_{1}\right)$,

$$
u\left(E_{\lambda} \cap \bigcup_{j} S_{j}^{1}\right) \leq C_{7} \sum_{j} u\left(S_{j}^{1}\right)=C_{7} u\left(\bigcup_{j} S_{j}^{1}\right) .
$$

This via (2.9) in turn implies that

$$
\begin{aligned}
u\left(E_{\lambda}\right) \leq & u\left(\left(\bigcup_{k=1}^{n_{B}} \bigcup_{j} S_{j}^{k}\right) \backslash \bigcup_{j} S_{j}^{1}\right)+u\left(E_{\lambda} \cap \bigcup_{j} S_{j}^{1}\right) \\
\leq & C_{7} u\left(\left(\bigcup_{k=1}^{n_{B}} \bigcup_{j} S_{j}^{k}\right) \backslash \bigcup_{j} S_{j}^{1}\right)+C_{7} u\left(\bigcup_{j} S_{j}^{1}\right) \\
& +\left(1-C_{7}\right) u\left(\left(\bigcup_{k=1}^{n_{B}} \bigcup_{j} S_{j}^{k}\right) \backslash \bigcup_{j} S_{j}^{1}\right) \\
\leq & C_{7} u\left(\bigcup_{k=1}^{n_{B}} \bigcup_{j} S_{j}^{k}\right)+\left(1-C_{7}\right)\left(1-\frac{1}{n_{B}}\right) u\left(\bigcup_{k=1}^{n_{B}} \bigcup_{j} S_{j}^{k}\right) \\
\leq & \left(1-\frac{1-C_{7}}{n_{B}}\right) u\left(\Omega_{\lambda}\right),
\end{aligned}
$$

which gives us the desired conclusion with $\theta_{1}=1-\left(1-C_{7}\right) / n_{B}$. 
We now prove (2.10). For each fixed $y \in S_{j}^{1} \cap E_{\lambda}$, we claim that if $Q$ is a doubling cube containing $y$ and satisfying $m_{0, s_{1} ; Q}^{\varrho}(f)>(1+\gamma) \lambda$, then $l(Q) \leq l\left(S_{j}^{1}\right) / 8$. Otherwise, $Q_{x_{j}^{1}} \subset S_{j}^{1} \subset \widetilde{30 Q}$, and so

$$
\begin{aligned}
\left|m_{0, s_{1} ; Q}^{\varrho}(f)-m_{0, s_{1} ; \widetilde{30 Q}}^{\varrho}(f)\right| & \leq 3 \delta_{Q, \widetilde{30 Q}}^{\varrho} M_{0, s_{1}}^{\varrho, \sharp} f(y) \\
& \leq 3 \delta_{Q, \widetilde{30 Q}}^{\varrho} M_{0, s_{2}}^{\varrho, \sharp} f(y) \\
& \leq C_{8} \theta_{2} \gamma \lambda,
\end{aligned}
$$

where $C_{8}>1$ is such that $3 \delta_{Q, \widetilde{30 Q}}^{\varrho} \leq C_{8}$; see Lemma 2.1 in [17]. Set $\theta_{2}=$ $1 /\left(2 C_{5}+2 C_{8}\right)$. It then follows that

$$
m_{0, s_{1} ; 30 Q}^{\varrho}(f)>(1+\gamma / 2) \lambda,
$$

which contradicts the fact that $Q_{x_{j}^{1}} \subset \widetilde{30 Q}, l(\widetilde{30 Q})>2 \varrho l\left(Q_{x_{j}^{1}}\right)$ and $Q_{x_{j}^{1}}$ is the chosen maximal doubling cube.

For each fixed $y \in S_{j}^{1} \cap E_{\lambda}$, we see that there exists a doubling cube $I$ such that $y \in I$ and $m_{0, s_{1} ; I}^{\varrho}(f)>(1+\gamma) \lambda$. Our claim then tells us that $l(I) \leq l\left(S_{j}^{1}\right)$ and $I \subset \frac{5}{4} S_{j}^{1}$. Thus,

$$
m_{0, s_{1} ; I}^{\varrho}\left(f \chi_{\frac{5}{4} S_{j}^{1}}\right)>(1+\gamma) \lambda .
$$

On the other hand, by Lemma 2.5 , we have

$$
\left|m_{f}\left(\widetilde{\frac{5}{4} S_{j}^{1}}\right)\right| \leq m_{0, s_{1} ; \frac{5}{4} S_{j}^{1}}^{\varrho}(f) \leq(1+\gamma / 2) \lambda .
$$

This via Lemma 2.4 gives

$$
m_{0, s_{1} ; I}^{\varrho}\left(\left(f-m_{f}\left(\widetilde{\frac{5}{4} S_{j}^{1}}\right)\right) \chi_{\frac{5}{4} S_{j}^{1}}\right)>\gamma \lambda / 2,
$$

and then

$$
S_{j}^{1} \cap E_{\lambda} \subset\left\{y \in \mathbb{R}^{d}: M_{0, s_{1}}^{\varrho, d}\left(\left(f-m_{f}\left(\widetilde{\frac{5}{4} S_{j}^{1}}\right)\right) \chi_{\frac{5}{4}} S_{j}^{1}\right)(y)>\gamma \lambda / 2\right\} .
$$

Invoking Lemma 2.3(ii) and the inequality (2.4) and noticing that $\theta_{2}<1 / 4$ by our choice, we find that for some $\sigma>0$,

$$
\begin{aligned}
\mu\left(S_{j}^{1} \cap E_{\lambda}\right) & \leq C s_{1}^{-1} \mu\left(\left\{y \in \frac{5}{4} S_{j}^{1}:\left|f(y)-m_{f}\left(\widetilde{\frac{5}{4} S_{j}^{1}}\right)\right|>\gamma \lambda / 2\right\}\right) \\
& \leq C s_{1}^{-1} \mu\left(\left\{y \in \frac{5}{4} S_{j}^{1}:\left|f(y)-m_{f}\left(\widetilde{\frac{5}{4} S_{j}^{1}}\right)\right|>2 M_{0, s_{2}}^{\varrho, \sharp} f\left(x_{j}^{1}\right)+\sigma\right\}\right) \\
& \leq C s_{1}^{-1} s_{2} \mu\left(2 \varrho S_{j}^{1}\right) \leq C s_{1}^{-1} s_{2} \mu\left(S_{j}^{1}\right),
\end{aligned}
$$

where $C$ is a positive constant. This finishes the proof of Lemma 2.6. 
Lemma 2.7. Let $\varrho \in[1, \infty)$ and $s \in\left(0, \beta_{2 \varrho, d}^{-1} / 4\right)$. Then for any $\mu$-locally integrable function $f$ and $x \in \mathbb{R}^{d}$,

$$
M_{0, s}^{\varrho, \sharp}(|f|)(x) \leq 8 M_{0, s}^{\varrho, \sharp}(f)(x) .
$$

Proof. Notice that for any cube $Q \subset \mathbb{R}^{d}, c \in \mathbb{C}$ and $\mu$-locally integrable function $h$,

$$
m_{h}(Q)-c=m_{h-c}(Q) .
$$

It follows from Lemmas 2.4 and 2.5 that

$$
\begin{aligned}
m_{0, s ; Q}^{\varrho}\left(|f|-m_{|f|}(\widetilde{Q})\right) & \leq m_{0, s ; Q}^{\varrho}\left(|f|-\left|m_{f}(\widetilde{Q})\right|\right)+|| m_{f}(\widetilde{Q})\left|-m_{|f|}(\widetilde{Q})\right| \\
& \leq m_{0, s ; Q}^{\varrho}\left(f-m_{f}(\widetilde{Q})\right)+\left|m_{|f|-\left|m_{f}(\widetilde{Q})\right|}(\widetilde{Q})\right| \\
& \leq m_{0, s ; Q}^{\varrho}\left(f-m_{f}(\widetilde{Q})\right)+2 m_{0, s ; \widetilde{Q}}^{\varrho}\left(|f|-\left|m_{f}(\widetilde{Q})\right|\right) \\
& \leq m_{0, s ; Q}^{\varrho}\left(f-m_{f}(\widetilde{Q})\right)+2 m_{0, s ; \widetilde{Q}}^{\varrho}\left(f-m_{f}(\widetilde{Q})\right) .
\end{aligned}
$$

On the other hand, by Lemma 2.5 again, we see that for any doubling cubes $Q \subset R$,

$$
\begin{aligned}
\mid m_{|f|} & (Q)-m_{|f|}(R) \mid \\
& \leq\left|m_{|f|}(Q)-\right| m_{f}(Q)||+|| m_{f}(Q)|-| m_{f}(R)||+\left|m_{|f|}(R)-\right| m_{f}(R)|| \\
& \leq\left|m_{|f|-\left|m_{f}(Q)\right|}(Q)\right|+\left|m_{f}(Q)-m_{f}(R)\right|+\left|m_{|f|-\left|m_{f}(R)\right|}(R)\right| \\
& \leq 2 m_{0, s ; Q}^{\varrho}\left(f-m_{f}(Q)\right)+\left|m_{f}(Q)-m_{f}(R)\right|+2 m_{0, s ; R}^{\varrho}\left(f-m_{f}(R)\right) .
\end{aligned}
$$

Combining the last two estimates then yields the desired estimate, which completes the proof of Lemma 2.7.

Proof of Theorem 2.1. By Lemma 2.7, we may assume that $f$ is realvalued. We first consider the case where $\mu\left(\mathbb{R}^{d}\right)=\infty$. By Lemma 2.6, we see that for any $\gamma>0$ and $\lambda>0$,

$$
\begin{aligned}
u(\{x \in & \left.\left.\mathbb{R}^{d}: M_{0, s_{1}}^{\varrho, d} f(x)>(1+\gamma) \lambda\right\}\right) \\
& \leq \theta_{1} u\left(\left\{x \in \mathbb{R}^{d}: M_{0, s_{1}}^{\varrho, d} f(x)>\lambda\right\}\right)+u\left(\left\{x \in \mathbb{R}^{d}: M_{0, s_{2}}^{\varrho, \sharp} f(x)>\theta_{2} \gamma \lambda\right\}\right),
\end{aligned}
$$

and consequently,

$$
\begin{aligned}
(1+\gamma)^{p} \lambda^{p} u\left(\left\{x \in \mathbb{R}^{d}:\right.\right. & \left.\left.M_{0, s_{1}}^{\varrho, d} f(x)>(1+\gamma) \lambda\right\}\right) \\
\leq & \theta_{1}(1+\gamma)^{p} \lambda^{p} u\left(\left\{x \in \mathbb{R}^{d}: M_{0, s_{1}}^{\varrho, d} f(x)>\lambda\right\}\right) \\
& +(1+\gamma)^{p} \lambda^{p} u\left(\left\{x \in \mathbb{R}^{d}: M_{0, s_{2}}^{\varrho, \sharp} f(x)>\theta_{2} \gamma \lambda\right\}\right) .
\end{aligned}
$$


Taking the supremum in the last inequality shows that for any $R>0$,

$$
\begin{aligned}
\sup _{0<\lambda<(1+\gamma) R} \lambda^{p} u(\{x & \left.\left.\in \mathbb{R}^{d}: M_{0, s_{1}}^{\varrho, d} f(x)>\lambda\right\}\right) \\
\leq & \theta_{1}(1+\gamma)^{p} \sup _{0<\lambda<R} \lambda^{p} u\left(\left\{x \in \mathbb{R}^{d}: M_{0, s_{1}}^{\varrho, d} f(x)>\lambda\right\}\right) \\
& +\left(\frac{1+\gamma}{\theta_{2} \gamma}\right)^{p} \sup _{\lambda>0} \lambda^{p} u\left(\left\{x \in \mathbb{R}^{d}: M_{0, s_{2}}^{\varrho, \sharp} f(x)>\lambda\right\}\right) .
\end{aligned}
$$

Notice that by Lemma 2.3,

$$
\begin{aligned}
\sup _{0<\lambda<R} \lambda^{p} u\left(\left\{x \in \mathbb{R}^{d}: M_{0, s_{1}}^{\varrho, d} f(x)\right.\right. & >\lambda\}) \\
& \leq C \sup _{0<\lambda<R} \lambda^{p} u\left(\left\{x \in \mathbb{R}^{d}:|f(x)|>\lambda\right\}\right),
\end{aligned}
$$

where $C$ is a positive constant. Our hypotheses guarantee that in this case,

$$
\sup _{0<\lambda<R} \lambda^{p} u\left(\left\{x \in \mathbb{R}^{d}: M_{0, s_{1}}^{\varrho, d} f(x)>\lambda\right\}\right)<\infty .
$$

Choosing $\gamma$ small enough such that $(1+\gamma)^{p} \theta_{1}<1$, we see that when $\mu\left(\mathbb{R}^{d}\right)=\infty$,

$$
\begin{aligned}
\sup _{0<\lambda<R} \lambda^{p} u\left(\left\{x \in \mathbb{R}^{d}: M_{0, s_{1}}^{\rho, d}\right.\right. & f(x)>\lambda\}) \\
& \leq C \sup _{0<\lambda<R} \lambda^{p} u\left(\left\{x \in \mathbb{R}^{d}: M_{0, s_{2}}^{\varrho, \sharp} f(x)>\lambda\right\}\right),
\end{aligned}
$$

where $C$ is a positive constant.

On the other hand, if $\mu\left(\mathbb{R}^{d}\right)<\infty$, another application of Lemma 2.6 indicates that for any $R>\lambda_{f}$ and $\gamma \in(0,1)$,

$$
\begin{aligned}
& \sup _{0<\lambda<(1+\gamma) R} \lambda^{p} u\left(\left\{x \in \mathbb{R}^{d}: M_{0, s_{1}}^{\varrho, d} f(x)>\lambda\right\}\right) \\
& \leq \quad \sup _{(1+\gamma) \lambda_{f} \leq \lambda<(1+\gamma) R} \lambda^{p} u\left(\left\{x \in \mathbb{R}^{d}: M_{0, s_{1}}^{\varrho, d} f(x)>\lambda\right\}\right) \\
& \quad+\sup _{0<\lambda<(1+\gamma) \lambda_{f}} \lambda^{p} u\left(\left\{x \in \mathbb{R}^{d}: M_{0, s_{1}}^{\varrho, d} f(x)>\lambda\right\}\right) \\
& \leq \quad(1+\gamma)^{p} \sup _{\lambda_{f} \leq \lambda<R} \lambda^{p} u\left(\left\{x \in \mathbb{R}^{d}: M_{0, s_{1}}^{\varrho, d} f(x)>(1+\gamma) \lambda\right\}\right) \\
& \quad+(1+\gamma)^{p} \lambda_{f}^{p} u\left(\mathbb{R}^{d}\right) \\
& \leq \quad(1+\gamma)^{p} \theta_{1} \sup _{\lambda_{f} \leq \lambda<R} \lambda^{p} u\left(\left\{x \in \mathbb{R}^{d}: M_{0, s_{1}}^{\varrho, d} f(x)>\lambda\right\}\right) \\
& \quad+\left(\frac{1+\gamma}{\theta_{2} \gamma}\right)^{p} \sup _{\lambda>0} \lambda^{p} u\left(\left\{x \in \mathbb{R}^{d}: M_{0, s_{2}}^{\varrho, \sharp} f(x)>\lambda\right\}\right)+(1+\gamma)^{p} \lambda_{f}^{p} u\left(\mathbb{R}^{d}\right) .
\end{aligned}
$$


Since $\mu\left(\mathbb{R}^{d}\right)<\infty$ implies $u\left(\mathbb{R}^{d}\right)<\infty$, we infer that when $\mu\left(\mathbb{R}^{d}\right)<\infty$,

$$
\begin{aligned}
\sup _{0<\lambda<R} \lambda^{p} u(\{x & \left.\left.\in \mathbb{R}^{d}: M_{0, s_{1}}^{\varrho, d} f(x)>\lambda\right\}\right) \\
\leq C & \sup _{0<\lambda<R} \lambda^{p} u\left(\left\{x \in \mathbb{R}^{d}: M_{0, s_{2}}^{\varrho, \sharp} f(x)>\lambda\right\}\right)+C \lambda_{f}^{p} u\left(\mathbb{R}^{d}\right),
\end{aligned}
$$

where $C$ is a positive constant. Taking $R \rightarrow \infty$ then leads to the desired conclusion (ii), which completes the proof of Theorem 2.1.

3. Proof of Theorem 1.1. By Lemma 2.1, it is obvious that Theorem 1.1 is an easy corollary of the following result.

TheOREM 3.1. Under the hypotheses of Theorem 1.1, for any $\varrho, p \in$ $[1, \infty)$ and $u \in A_{p}^{\varrho}(\mu)$, there exists a positive constant $C$ such that for any bounded function $f$ with compact support,

$$
\sup _{\lambda>0} \lambda^{p} u\left(\left\{x \in \mathbb{R}^{d}: T^{*} f(x)>\lambda\right\}\right) \leq C \sup _{\lambda>0} \lambda^{p} u\left(\left\{x \in \mathbb{R}^{d}: M_{\frac{9}{8} \varrho} f(x)>\lambda\right\}\right) .
$$

To prove Theorem 3.1, we begin with an inequality relating the sharp maximal operator $M_{0, s}^{\varrho, \sharp}$ and a variant of the sharp maximal operator of Tolsa [17]. Let $r \in(0, \infty)$. Define the sharp maximal operator $M_{r}^{\varrho, \sharp}$ by setting, for all $x \in \mathbb{R}^{d}$,

$$
\begin{aligned}
M_{r}^{\varrho, \sharp} f(x)= & \sup _{x \in Q}\left(\frac{1}{\mu\left(\frac{3}{2} \varrho Q\right)} \int_{Q}\left|f(x)-m_{f}(\widetilde{Q})\right|^{r} d \mu(x)\right)^{1 / r} \\
& +\sup _{\substack{x \in Q \subset R \\
Q, R \text { doubling }}} \frac{\left|m_{f}(Q)-m_{f}(R)\right|}{\delta_{Q, R}^{\varrho}} .
\end{aligned}
$$

For $r=1$ and $\varrho=1$, this operator is the sharp maximal operator introduced by Tolsa in [17]. It is obvious that for any cube $Q$ and $r \in(0, \infty)$,

$$
m_{0, s ; Q}^{\varrho}\left(f-m_{f}(\widetilde{Q})\right) \leq s^{-1 / r}\left(\frac{1}{\mu\left(\frac{3}{2} \varrho Q\right)} \int_{Q}\left|f(y)-m_{f}(\widetilde{Q})\right|^{r} d \mu(y)\right)^{1 / r} .
$$

Therefore, for all $x \in \mathbb{R}^{d}$,

$$
M_{0, s}^{\varrho, \sharp} f(x) \leq s^{-1 / r} M_{r}^{\varrho, \sharp} f(x) .
$$

Moreover, we have the following several technical lemmas.

Lemma 3.1. Let $\varrho \in[1, \infty), s \in\left(0, \beta_{2 \varrho, d}^{-1} / 4\right)$ and $r \in(0, \infty)$. For any cube $Q$ and $\mu$-locally integrable function $f$,

$$
m_{0, s ; Q}^{\varrho}\left(f-m_{f}(Q)\right) \leq 3 s^{-1 / r} \inf _{c \in \mathbb{C}}\left(\frac{1}{\mu\left(\frac{3}{2} \varrho Q\right)} \int_{Q}|f(y)-c|^{r} d \mu(y)\right)^{1 / r} .
$$


Proof. For any cube $Q$ and $\mu$-locally integrable function $f$, set

$$
m_{0, s ; Q}^{\varrho, \sharp}(f)=\inf _{c \in \mathbb{C}} m_{0, s ; Q}^{\varrho}(f-c) .
$$

Then, to prove Lemma 3.1, it suffices to prove that

$$
m_{0, s ; Q}^{\varrho}\left(f-m_{f}(Q)\right) \leq 3 m_{0, s ; Q}^{\varrho, \sharp}(f) .
$$

The estimate (3.2) is trivial if $\mu(Q)=0$; so we need only consider the case where $\mu(Q)>0$. For any fixed $\varepsilon>0$, we choose $c_{Q}=a+i b$ such that

$$
\mu\left(\left\{y \in Q:\left|f(y)-c_{Q}\right|>m_{0, s ; Q}^{\varrho, \sharp}(f)+\varepsilon\right\}\right)<s \mu\left(\frac{3}{2} \varrho Q\right) .
$$

Thus,

$$
\begin{aligned}
& \mu\left(\left\{y \in Q:|\operatorname{Re} f(y)-a|>m_{0, s ; Q}^{\varrho, \sharp}(f)+\varepsilon\right\}\right)<s \mu\left(\frac{3}{2} \varrho Q\right), \\
& \mu\left(\left\{y \in Q:|\operatorname{Im} f(y)-b|>m_{0, s ; Q}^{\varrho, \sharp}(f)+\varepsilon\right\}\right)<s \mu\left(\frac{3}{2} \varrho Q\right) .
\end{aligned}
$$

If $\operatorname{Re} m_{f}(Q)>a+m_{0, s ; Q}^{\varrho, \sharp}(f)+\varepsilon$, then by (3.3),

$$
\begin{aligned}
\mu(\{y \in Q: \operatorname{Re} f(y)<\operatorname{Re} & \left.\left.m_{f}(Q)\right\}\right) \\
& \geq \mu\left(\left\{y \in Q: \operatorname{Re} f(y) \leq a+m_{0, s ; Q}^{\varrho, \sharp}(f)+\varepsilon\right\}\right) \\
& \geq\left(1-s \beta_{2 \varrho, d}\right) \mu(Q)>\frac{1}{2} \mu(Q),
\end{aligned}
$$

which contradics the definition of $\operatorname{Re} m_{f}(Q)$. Therefore,

$$
\operatorname{Re} m_{f}(Q) \leq a+m_{0, s ; Q}^{\varrho, \sharp}(f)+\varepsilon .
$$

We can also deduce from (3.3) that

$$
\operatorname{Re} m_{f}(Q) \geq a-m_{0, s ; Q}^{\varrho, \sharp}(f)-\varepsilon,
$$

and from (3.4) that

$$
b-m_{0, s ; Q}^{\varrho, \sharp}(f)-\varepsilon \leq \operatorname{Im} m_{f}(Q) \leq b+m_{0, s ; Q}^{\varrho, \sharp}(f)+\varepsilon .
$$

Combining these estimates yields

$$
\left|m_{f}(Q)-c_{Q}\right| \leq 2 m_{0, s ; Q}^{\varrho, \sharp}(f)+2 \varepsilon,
$$

which leads to

$$
\begin{aligned}
\left\{y \in Q:\left|f(y)-m_{f}(Q)\right|>\right. & \left.3 m_{0, s ; Q}^{\varrho, \sharp}(f)+3 \varepsilon\right\} \\
& \subset\left\{y \in Q:\left|f(y)-c_{Q}\right|>m_{0, s ; Q}^{\varrho, \sharp}(f)+\varepsilon\right\} .
\end{aligned}
$$

We then obtain

$$
\mu\left(\left\{y \in Q:\left|f(y)-m_{f}(Q)\right|>3 m_{0, s ; Q}^{\varrho, \sharp}(f)+3 \varepsilon\right\}\right)<s \mu\left(\frac{3}{2} \varrho Q\right),
$$

and so

$$
m_{0, s ; Q}^{\varrho}\left(f-m_{f}(Q)\right) \leq 3 m_{0, s ; Q}^{\varrho, \sharp}(f)+3 \varepsilon .
$$

The inequality (3.2) then follows by letting $\varepsilon \rightarrow 0$, which completes the proof of Lemma 3.1 . 
Lemma 3.2. Let $\varrho \in[1, \infty)$ and $r \in(0,1)$. Under the assumptions of Theorem 1.1, there exists a positive constant $C$ such that for all bounded functions $f$ with compact support and $x \in \mathbb{R}^{d}$,

$$
M_{r}^{\varrho, \sharp}\left(T^{*} f\right)(x) \leq C M_{\frac{9}{8} \varrho} f(x) .
$$

Proof. For each cube $Q$ and each bounded function $f$ with compact support, set

$$
h_{Q}=m_{Q}\left(T^{*}\left(f \chi_{\mathbb{R}^{d} \backslash \frac{4}{3} Q}\right)\right) ;
$$

here and in what follows, for any $\mu$-locally integrable function $h$,

$$
m_{Q}(h)=\frac{1}{\mu(Q)} \int_{Q} h(z) d \mu(z) .
$$

It follows from Lemmas 2.4 and 3.1 that for any cube $Q$ and $s \in\left(0, \beta_{2 \varrho, d}^{-1} / 4\right)$,

$$
\begin{aligned}
\int_{Q} \mid T^{*} f(y)- & \left.m_{T^{*} f}(\widetilde{Q})\right|^{r} d \mu(y) \leq \int_{Q}\left|T^{*} f(y)-h_{Q}\right|^{r} d \mu(y)+\left|h_{Q}-h_{\widetilde{Q}}\right|^{r} \mu(Q) \\
& +\left|m_{0, s ; \widetilde{Q}}^{\varrho}\left(T^{*} f\right)-m_{T^{*} f}(\widetilde{Q})\right|^{r} \mu(Q)+\left|m_{0, s ; \widetilde{Q}}^{\varrho}\left(T^{*} f\right)-h_{\widetilde{Q}}\right|^{r} \mu(Q) \\
\leq & \int_{Q}\left|T^{*} f(y)-h_{Q}\right|^{r} d \mu(y)+\left|h_{Q}-h_{\widetilde{Q}}\right|^{r} \mu(Q) \\
& +\left(m_{0, s ; \widetilde{Q}}^{\varrho}\left(T^{*} f-m_{T^{*} f}(\widetilde{Q})\right)\right)^{r} \mu(Q)+\left(m_{0, s ; \widetilde{Q}}^{\varrho}\left(T^{*} f-h_{\widetilde{Q}}\right)\right)^{r} \mu(Q) \\
\leq & \int_{Q}\left|T^{*} f(y)-h_{Q}\right|^{r} d \mu(y)+\left|h_{Q}-h_{\widetilde{Q}}\right|^{r} \mu(Q) \\
& +C\left(3^{r} s^{-1}+s^{-1}\right) \frac{\mu(Q)}{\mu(\widetilde{Q})} \int_{\widetilde{Q}}\left|T^{*} f(y)-h_{\widetilde{Q}}\right|^{r} d \mu(y),
\end{aligned}
$$

where $C$ is a positive constant, and for any two doubling cubes $Q \subset R$,

$$
\begin{aligned}
\mid m_{T^{*} f}(Q)- & m_{T^{*} f}(R) \mid \\
\leq & \left|m_{0, s ; Q}^{\varrho}\left(T^{*} f\right)-h_{Q}\right|+\left|h_{Q}-h_{R}\right|+\left|m_{0, s ; R}^{\varrho}\left(T^{*} f\right)-h_{R}\right| \\
& \quad+\left|m_{0, s ; Q}^{\varrho}\left(T^{*} f\right)-m_{T^{*} f}(Q)\right|+\left|m_{0, s ; R}^{\varrho}\left(T^{*} f\right)-m_{T^{*} f}(R)\right| \\
\leq & m_{0, s ; Q}^{\varrho}\left(T^{*} f-h_{Q}\right)+\left|h_{Q}-h_{R}\right|+m_{0, s ; R}^{\varrho}\left(T^{*} f-h_{R}\right) \\
& \quad+m_{0, s ; Q}^{\varrho}\left(T^{*} f-m_{T^{*} f}(Q)\right)+m_{0, s ; R}^{\varrho}\left(T^{*} f-m_{T^{*} f}(R)\right) \\
\leq & 4 s^{-1 / r}\left(\frac{1}{\mu\left(\frac{3}{2} \varrho Q\right)} \int_{Q}\left|T^{*} f(y)-h_{Q}\right|^{r} d \mu(y)\right)^{1 / r}+\left|h_{Q}-h_{R}\right| \\
& \quad+4 s^{-1 / r}\left(\frac{1}{\mu\left(\frac{3}{2} \varrho R\right)} \int_{R}\left|T^{*} f(y)-h_{R}\right|^{r} d \mu(y)\right)^{1 / r} .
\end{aligned}
$$


Thus, the proof of (3.5) can be reduced to proving that for any cube $Q$,

$$
\left(\frac{1}{\mu\left(\frac{3}{2} \varrho Q\right)} \int_{Q}\left|T^{*} f(y)-h_{Q}\right|^{r} d \mu(y)\right)^{1 / r} \leq C \inf _{x \in Q} M_{\frac{9}{8} \varrho} f(x),
$$

and for any two cubes $Q \subset R$ with $R$ a doubling cube,

$$
\left|h_{Q}-h_{R}\right| \leq C \delta_{Q, R}^{\varrho} \inf _{x \in Q} M_{\frac{9}{8} \varrho} f(x),
$$

where $C$ is a positive constant.

To prove (3.6) and (3.7), we employ the argument used in [2]. We first consider (3.6). For any cube $Q$, write

$$
\begin{aligned}
\int_{Q}\left|T^{*} f(y)-h_{Q}\right|^{r} d \mu(y) \leq & \int_{Q}\left|T^{*} f(y)-T^{*}\left(f \chi_{\mathbb{R}^{d} \backslash \frac{4}{3} Q}\right)(y)\right|^{r} d \mu(y) \\
& +\int_{Q}\left|T^{*}\left(f \chi_{\mathbb{R}^{d} \backslash \frac{4}{3} Q}\right)(y)-h_{Q}\right|^{r} d \mu(y) \\
\leq & \int_{Q}\left|T^{*}\left(f \chi_{\frac{4}{3} Q}\right)(y)\right|^{r} d \mu(y) \\
& +\int_{Q}\left|T^{*}\left(f \chi_{\mathbb{R}^{d} \backslash \frac{4}{3} Q}\right)(y)-h_{Q}\right|^{r} d \mu(y) .
\end{aligned}
$$

Recall that $T^{*}$ is bounded from $L^{1}(\mu)$ to weak $L^{1}(\mu)$; see [11] or [2]. It follows from the Kolmogorov inequality that

$$
\begin{aligned}
\left(\frac{1}{\mu\left(\frac{3}{2} \varrho Q\right)} \int_{Q}\left|T^{*}\left(f \chi_{\frac{4}{3} Q}\right)(y)\right|^{r} d \mu(y)\right)^{1 / r} & \leq \frac{C}{\mu\left(\frac{3}{2} \varrho Q\right)}\left\|f \chi_{\frac{4}{3} Q}\right\|_{L^{1}(\mu)} \\
& \leq C \inf _{x \in Q} M_{\frac{9}{8} \varrho} f(x),
\end{aligned}
$$

where $C$ is a positive constant. On the other hand, the size condition (1.2) and the regularity condition (1.3) via a standard computation imply that for any $y, z \in Q$,

$$
\begin{aligned}
\mid T^{*}\left(f \chi_{\mathbb{R}^{d} \backslash \frac{4}{3} Q}\right)(y)- & T^{*}\left(f \chi_{\mathbb{R}^{d} \backslash \frac{4}{3} Q}\right)(z) \mid \\
\leq & \sup _{\varepsilon>0} \mid \int_{|y-w|>\varepsilon} K(y, w) f(w) \chi_{\mathbb{R}^{d} \backslash \frac{4}{3} Q}(w) d \mu(w) \\
& -\int_{|y-w|>\varepsilon} K(z, w) f(w) \chi_{\mathbb{R}^{d} \backslash \frac{4}{3} Q}(w) d \mu(w) \mid \\
& +\sup _{\varepsilon>0} \mid \int_{|y-w|>\varepsilon} K(z, w) f(w) \chi_{\mathbb{R}^{d} \backslash \frac{4}{3} Q}(w) d \mu(w) \\
& -\int_{|z-w|>\varepsilon} K(z, w) f(w) \chi_{\mathbb{R}^{d} \backslash \frac{4}{3} Q}(w) d \mu(w) \mid
\end{aligned}
$$




$$
\begin{aligned}
& \leq \int_{\mathbb{R}^{d} \backslash \frac{4}{3} Q}|K(y, w)-K(z, w)||f(w)| d \mu(w) \\
& \quad+C \sup _{\varepsilon>0} \varepsilon^{-n} \int_{|z-w|<C \varepsilon}|f(w)| \chi_{\mathbb{R}^{d} \backslash \frac{4}{3} Q}(w) d \mu(w) \\
& \leq C \inf _{x \in Q} M_{\frac{9}{8} \varrho} f(x),
\end{aligned}
$$

where $C$ is a positive constant. This in turn implies that for a positive constant $C$,

$$
\left\{\frac{1}{\mu\left(\frac{3}{2} \varrho Q\right)} \int_{Q}\left|T^{*}\left(f \chi_{\mathbb{R}^{d} \backslash \frac{4}{3} Q}\right)(y)-h_{Q}\right|^{r} d \mu(y)\right\}^{1 / r} \leq C \inf _{x \in Q} M_{\frac{9}{8} \varrho} f(x),
$$

which gives the estimate (3.6).

Now we turn to (3.7). Denote $N_{Q, R}^{\varrho}+1$ simply by $N$. Write

$$
\begin{aligned}
\left|h_{Q}-h_{R}\right| \leq & m_{Q}\left(T^{*}\left(f \chi_{2 \varrho Q \backslash \frac{4}{3} Q}\right)\right)+m_{Q}\left(T^{*}\left(f \chi_{(2 \varrho)^{N} Q \backslash 2 \varrho Q}\right)\right) \\
& +\left|m_{Q}\left(T^{*}\left(f \chi_{\mathbb{R}^{d} \backslash(2 \varrho)^{N} Q}\right)\right)-m_{R}\left(T^{*}\left(f \chi_{\mathbb{R}^{d} \backslash(2 \varrho)^{N} Q}\right)\right)\right| \\
& +m_{R}\left(T^{*}\left(f \chi_{(2 \varrho)^{N} Q \backslash \frac{4}{3} R}\right)\right) \\
= & \mathrm{I}_{1}+\mathrm{I}_{2}+\mathrm{I}_{3}+\mathrm{I}_{4} .
\end{aligned}
$$

The size condition (1.2) along with the growth condition (1.1) implies that for any $x, y \in Q$,

$$
T^{*}\left(f \chi_{2 \varrho Q \backslash \frac{4}{3} Q}\right)(y) \leq \frac{C}{[l(Q)]^{n}} \int_{2 \varrho Q}|f(z)| d \mu(z) \leq C M_{\frac{9}{8} \varrho} f(x),
$$

and for any $y \in R$ and $x \in Q$,

$$
T^{*}\left(f \chi_{(2 \varrho)^{N} Q \backslash \frac{4}{3} R}\right)(y) \leq \frac{C}{[l(R)]^{n}} \int_{4 \varrho R}|f(z)| d \mu(z) \leq C M_{\frac{9}{8} \varrho} f(x),
$$

where $C$ is a positive constant. Therefore, for a positive constant $C$,

$$
\mathrm{I}_{1}+\mathrm{I}_{4} \leq C \inf _{x \in Q} M_{\frac{9}{8}} f(x) .
$$

For the term $\mathrm{I}_{2}$, observing that for any $y \in Q$,

$$
\begin{aligned}
T^{*}\left(f \chi_{(2 \varrho)^{N} Q \backslash 2 \varrho Q}\right)(y) & \leq C \sum_{k=1}^{N-1} \int_{(2 \varrho)^{k+1} Q \backslash(2 \varrho)^{k} Q} \frac{|f(z)|}{|y-z|^{n}} d \mu(z) \\
& \leq C \sum_{k=1}^{N-1} \frac{\mu\left((2 \varrho)^{k+2} Q\right)}{\left[l\left((2 \varrho)^{k} Q\right)\right]^{n}} \inf _{x \in Q} M_{2 \varrho} f(x) \\
& \leq C \delta_{Q, R}^{\varrho} \inf _{x \in Q} M_{\frac{9}{8} \varrho} f(x)
\end{aligned}
$$


so we have

$$
\mathrm{I}_{2} \leq C \delta_{Q, R}^{\varrho} \inf _{x \in Q} M_{\frac{9}{8} \varrho} f(x),
$$

where $C$ is a positive constant.

Finally, as in the inequality (3.8), a familiar argument involving the condition (1.3) gives, for any $y \in Q$ and $z \in R$,

$$
\left|T^{*}\left(f \chi_{\mathbb{R}^{d} \backslash(2 \varrho)^{N} Q}\right)(y)-T^{*}\left(f \chi_{\mathbb{R}^{d} \backslash(2 \varrho)^{N} Q}\right)(z)\right| \leq C \inf _{x \in Q} M_{\frac{9}{8} \varrho} f(x),
$$

and so

$$
\mathrm{I}_{3} \leq C \inf _{x \in Q} M_{\frac{9}{8} \varrho} f(x),
$$

where $C$ is a positive constant. The inequality (3.7) now follows, which completes the proof of Lemma 3.2.

Proof of Theorem 3.1. We first prove that if $p \in[1, \infty)$, then for any bounded function $f$ with compact support,

$$
T^{*} f \in L^{p, \infty}(\mu),
$$

and for any $\varrho, p \in[1, \infty)$ and $u \in A_{p}^{\varrho}(\mu)$,

$$
\sup _{0<\lambda<R} \lambda^{p} u\left(\left\{x \in \mathbb{R}^{d}:\left|T^{*} f(x)\right|>\lambda\right\}\right)<\infty .
$$

The fact (3.9) was proved in [11] and [2]. To prove (3.10), let $t>2$ be large enough such that the support of $f$ is contained in the ball $B(0, t)$. It is obvious that

$$
\sup _{0<\lambda<R} \lambda^{p} u\left(\left\{x \in B(0,2 t):\left|T^{*} f(x)\right|>\lambda\right\}\right) \leq R^{p} u(B(0,2 t))<\infty .
$$

On the other hand, it is easy to see that if $x \in \mathbb{R}^{d} \backslash B(0,2 t)$, then by the size condition (1.2),

$$
T^{*} f(x) \leq \int_{\mathbb{R}^{d}}|K(x, y) f(y)| d \mu(y) \leq \frac{C_{9}}{|x|^{n}}\|f\|_{L^{1}(\mu)} .
$$

This via Lemma 2.2(i) and the growth condition (1.1) implies that if $\lambda \leq$ $C_{9}\|f\|_{L^{1}(\mu)} / 2$,

$$
\begin{aligned}
u\left(\left\{x \in \mathbb{R}^{d} \backslash B(0,2 t)\right.\right. & \left.\left.:\left|T^{*} f(x)\right|>\lambda\right\}\right) \\
& \leq u\left(\left\{x \in \mathbb{R}^{d}:|x|^{-n}>\lambda /\left(C_{9}\|f\|_{L^{1}(\mu)}\right)\right\}\right) \\
& \leq u\left(B\left(0, \frac{9}{8} \varrho\left(C_{9}\|f\|_{L^{1}(\mu)}\right)^{1 / n} \lambda^{-1 / n}\right)\right) \\
& \leq C u(B(0,1))\left(\frac{\mu\left(B\left(0, \frac{9}{8} \varrho C_{9}\|f\|_{L^{1}(\mu)}^{1 / n} \lambda^{-1 / n}\right)\right)}{\mu(B(0,1))}\right)^{p} \\
& \leq C_{f} \frac{u(B(0,1))}{[\mu(B(0,1))]^{p}} \lambda^{-p},
\end{aligned}
$$

where $C_{f}$ is a positive constant. 
Notice that for $\lambda>C_{9}\|f\|_{L^{1}(\mu)} / 2$, there exists no point $x \in \mathbb{R}^{d} \backslash B(0,2 t)$ satisfying $T^{*} f(x)>\lambda$. Therefore,

$$
\begin{aligned}
\sup _{\lambda>0} \lambda^{p} u(\{x & \left.\left.\in \mathbb{R}^{d} \backslash B(0,2 t):\left|T^{*} f(x)\right|>\lambda\right\}\right) \\
& =\sup _{0<\lambda \leq C_{9}\|f\|_{L^{1}(\mu)} / 2} \lambda^{p} u\left(\left\{x \in \mathbb{R}^{d} \backslash B(0,2 t):\left|T^{*} f(x)\right|>\lambda\right\}\right) \\
& \leq C_{f} \frac{u(B(0,1))}{[\mu(B(0,1))]^{p}},
\end{aligned}
$$

which proves (3.10).

We can now conclude the proof of Theorem 3.1. If $\mu\left(\mathbb{R}^{d}\right)=\infty$, the desired result follows from Lemma 2.3(i), Theorem 2.1 with $s_{1}=\beta_{2 \varrho, d}^{-1} / 5$ and $p_{0}=1,(3.1)$ and Lemma 3.2.

If $\mu\left(\mathbb{R}^{d}\right)<\infty, \varrho, p \in[1, \infty)$ and $u \in A_{p}^{\varrho}(\mu)$, then for a positive constant $C$,

$$
\begin{aligned}
u\left(\mathbb{R}^{d}\right)\left[\mu\left(\mathbb{R}^{d}\right)\right]^{-p}\left\|T^{*} f\right\|_{L^{1, \infty}(\mu)}^{p} & \leq C u\left(\mathbb{R}^{d}\right)\left[\mu\left(\mathbb{R}^{d}\right)\right]^{-p}\|f\|_{L^{1}(\mu)}^{p} \\
& \leq C u\left(\mathbb{R}^{d}\right)\left(\inf _{x \in \mathbb{R}^{d}} M_{\frac{9}{8} \varrho} f(x)\right)^{p} \\
& \leq C \sup _{\lambda>0}\left[\lambda^{p} u\left(\left\{x \in \mathbb{R}^{d}: M_{\frac{9}{8} \varrho} f(x)>\lambda\right\}\right)\right],
\end{aligned}
$$

where in the first inequality, we have invoked the estimate

$$
\left\|T^{*} f\right\|_{L^{1, \infty}(\mu)} \leq C\|f\|_{L^{1}(\mu)}
$$

(see [11] or [2]), and the second inequality follows from the fact that

$$
\frac{1}{\mu\left(\mathbb{R}^{d}\right)} \int_{\mathbb{R}^{d}}|f(y)| d \mu(y)=\lim _{l(Q) \rightarrow \infty} \frac{1}{\mu\left(\frac{9}{8} \varrho Q\right)} \int_{Q}|f(y)| d \mu(y) \leq \inf _{x \in \mathbb{R}^{d}} M_{\frac{9}{8} \varrho} f(x) .
$$

The desired result again follows from Lemma 2.3(i), Theorem 2.1 with $s_{1}=$ $\beta_{2 \varrho, d}^{-1} / 5$ and $p_{0}=1,(3.1)$ and Lemma 3.2, which completes the proof of Theorem 3.1.

\section{References}

[1] G. E. Hu, Y. Meng and D. C. Yang, New atomic characterization of $H^{1}$ space with non-doubling measures and its applications, Math. Proc. Cambridge Philos. Soc. 138 (2005), 151-171.

[2] - , - - Estimates for maximal singular integral operators in non-homogeneous spaces, Proc. Roy. Soc. Edinburgh Ser. A 136 (2006), 351-364.

[3] G. E. Hu, X. Wang and D. C. Yang, A new characterization of regular BMO with non-doubling measures, Proc. Edinburgh Math. Soc. 51 (2008), 155-170.

[4] B. Jawerth and A. Torchinsky, Local sharp maximal functions, J. Approx. Theory 43 (1985), 231-270.

[5] F. John, Quasi-isometric mappings, in: Seminari 1962/63 Anal. Alg. Geom. e Topol., vol. 2, Ist. Naz. Alta Mat., Ediz. Cremonese, Rome, 1965, 462-473. 
[6] Y. Komori, Weighted estimates for operators generated by maximal functions on nonhomogeneous spaces, Georgian Math. J. 12 (2005), 121-130.

[7] A. K. Lerner, Weighted norm inequalities for the local sharp maximal function, J. Fourier Anal. Appl. 10 (2004), 465-474.

[8] —, Weighted rearrangement inequalities for local sharp maximal functions, Trans. Amer. Math. Soc. 357 (2005), 2445-2465.

[9] J. Mateu, P. Mattila, A. Nicolau and J. Orobitg, BMO for nondoubling measures, Duke Math. J. 102 (2000), 533-565.

[10] F. Nazarov, S. Treil and A. Volberg, Cauchy integral and Calderón-Zygmund operators on nonhomogeneous spaces, Int. Math. Res. Notices 1997, no. 15, 703-726.

[11] - , 一, 一, Weak type estimates and Cotlar inequalities for Calderón-Zygmund operators on nonhomogeneous spaces, ibid. 1998, no. 9, 463-487.

[12] - , - - Accretive system Tb-theorems on nonhomogeneous spaces, Duke Math. J. 113 (2002), 259-312.

[13] J. Orobitg and C. Pérez, $A_{p}$ weights for nondoubling measures in $\mathbb{R}^{n}$ and applications, Trans. Amer. Math. Soc. 354 (2002), 2013-2033.

[14] E. M. Stein, Harmonic Analysis: Real-Variable Methods, Orthogonality, and Oscillatory Integrals, Princeton Univ. Press, Princeton, NJ, 1993.

[15] J. O. Strömberg, Bounded mean oscillation with Orlicz norms and duality of Hardy spaces, Indiana Univ. Math. J. 28 (1979), 511-544.

[16] X. Tolsa, A proof of weak $(1,1)$ inequality for singular integrals with non doubling measures based on a Calderón-Zygmund decomposition, Publ. Mat. Barcelona 45 (2001), 163-174.

[17] - BMO, $H^{1}$ and Calderón-Zygmund operators for non doubling measures, Math. Ann. 319 (2001), 89-149.

[18] - The space $H^{1}$ for nondoubling measures in terms of a grand maximal operator, Trans. Amer. Math. Soc. 355 (2003), 315-348.

[19] —, Painlevé's problem and the semiadditivity of analytic capacity, Acta Math. 190 (2003), 105-149.

[20] J. Verdera, The fall of the doubling condition in Calderón-Zygmund theory, Publ. Mat. Barcelona 2002, special vol., 275-292.

Department of Applied Mathematics

University of Information Engineering

P.O. Box 1001-747

Zhengzhou 450002

People's Republic of China

E-mail: guoenxx@yahoo.com.cn
School of Mathematical Sciences Beijing Normal University Laboratory of Mathematics and Complex Systems Ministry of Education Beijing 100875

People's Republic of China E-mail: dcyang@bnu.edu.cn 